\title{
Enhancing the Bitrate and Power Spectral Density of PPM TH-IR UWB Signals using a Sub-Slot Technique
}

\author{
Bashar Al-haj Moh'd ${ }^{1}$ \\ Department of Medical Engineering \\ Al-Ahliyya Amman University \\ Amman, Jordan
}

\author{
Nidal Qasem ${ }^{2}$ \\ Department of Electronics and Communications \\ Engineering, Al-Ahliyya Amman University \\ Amman, Jordan
}

\begin{abstract}
Increasing the receiver's bitrate and suppressing the spectral line are issues of major interest in the design of compliant Time-Hopping Impulse Radio (TH-IR) Ultra-Wide Band (UWB) systems. Suppression of spectral lines has been commonly addressed by randomizing the position of each pulse to make the period as large as possible. Our analysis suggests that this influences the overall shape of a signal's Power Spectral Density (PSD) in a way that is useful for spectral line suppression or diminishing the PSD maximum peak power. A method for utilizing the system to generate a Dynamic-Location PulsePosition Modulated (DLPPM) signal for transmission across a UWB communications channel is presented, and an analytical derivation of the PSD of a proposed DLPPM signal TH-IR UWB is introduced. Our proposed method can be applied without affecting the users of other concurrent applications. The theoretical model for DPLM TH-IR is compared with the PSD for conventional DPLM TH-IR. The results show that spectral estimation methods based on Fast Fourier Transform (FFT) significantly overestimate the continuous part of the PSD for small and medium signal lengths, which has implications for assessing interference margins by means of simulation. Another purpose of this paper is to improve a predesigned system by increasing the receiver's bitrate. This will be achieved by using the bits that control the sub-slot technique as information and designing a receiver capable of detecting them. The bitrate is effectively doubled. Finally, the proposed system for DPLM THIR has been built inside Simulink/MATLAB to test its results via a conventional DPLM TH-IR system.
\end{abstract}

Keywords-Bitrate; FFT; PPM; PSD; spectral estimation; subslot; TH-IR; UWB

\section{INTRODUCTION}

Ultra-Wide Band (UWB) systems based on Impulse Radio (IR) are the leading candidates for communication systems with low power, low complexity, low rate, and high battery life, as well as immunity to multipath interference characteristics. Applications involving such systems range from collision avoidance automotive systems to sensor networks. IR-UWB technology (also referred to as impulse, baseband, and zero-carrier technology), uses very short pulses, which imply a large signal bandwidth, to convey information [1]. Various modulation techniques, such as Pulse-Amplitude Modulation (PAM), Pulse-Interval Modulation (PIM), PulseShape Modulation (PSM), Pulse-Position Modulation (PPM), On-Off Keying (OOK), and Bi-Phase Shift Keying (BPSK), are used to transmit the information in such systems [2, 3]. In PPM, the information is determined by the position of one pulse [4].

In order to be able to deploy such applications, the interference from UWB-based devices to already-established narrowband deployments must be kept to satisfactory levels. Consequently, the Power Spectral Density (PSD) of IR-UWBbased devices must comply with regulatory spectral masks such as the one used by the Federal Communications Commission (FCC) [5]. In this context, simulation of the signals produced by UWB devices with their corresponding PSD estimation by Fast Fourier Transform (FFT) methods is an invaluable tool for the evaluation and improvement of such systems before building the physical prototype.

Conventional PPM TH-IR typically assumes a fixed timing offset between pulses in the signal set. This has several drawbacks for UWB systems. Therefore, there is a need for modulation schemes to realize the benefits of standard PPM while providing greater randomness.

Thus, a novel PPM scheme must be more robust to fix timing offset effects while allowing for a greater throughput in UWB systems than conventional PPM TH-IR schemes. Therefore, methods and systems for generating DynamicLocation Pulse-Position Modulation (DLPPM) have been introduced [6].

In previous research $[1,7,8]$, the behaviour of simulations with FFT-based PSD estimation of UWB signals was analysed by comparing the results with analytical and actual measurements. This paper is an extension of such works, whereby a comparison between the proposed and existing systems is introduced.

The use of FFT-periodogram estimation methods for spectrum analysis of random signals is well studied in the literature [9]. However, some constraints must be observed when using these methods for the purpose of assessing, via simulation, the PSD behaviour of a particular UWB system before implementation. In this work, the behaviour of such estimation methods for proposed DLPPM TH-IR as a function of the sample length is analysed by comparing with previous results of conventional DLPPM TH-IR and PPM TH-IR [1,6] obtained with a swept spectrum analyser. This allows the 
identification of several issues that must be considered to enhance the PSD of conventional PPM TH-IR UWB signals. The proposed DLPPM TH-IR utilised the subsections to double the bitrate in comparison with conventional DLPPM TH-IR, but the PSD is not affected.

This paper is organised as follows: Section 2 describes the basic system properties and derivation of the conventional and proposed DLPPM TH-IR UWB schemes. Section 3 shows analytical and simulation results of the described system performance, and the conclusions are given in Section 4.

\section{SignAL REPRESENTATION AND ANALYTICAL PSD OF A DLPPM TH-IR SYSTEM}

\section{A. Conventional DLPPM TH-IR System}

Before discussing issues related to estimation of the spectrum power and increasing the bitrate for the proposed DLPPM TH-IR, we introduce the signal considered for analysing the proposed DLPPM TH-IR UWB system. The block diagram shown in Fig. 1 consists of six stages and manages to diminish the problem of the spectral lines and obtain a smooth PSD by observing the output at the spectrum analyzer [6].

The signal generated by the conventional DLPPM TH-IR system, shown in Fig. 1, is described by the formula [6]:

$x(t)=\sum_{m} w\left(t-m T_{r}-\left(\beta_{m}+\frac{\alpha_{m}}{N_{s S}}\right) T_{B}\right)$

where $w(t)$ accounts for the pulse shape, $T_{r}$ is the mean pulse repetition rate, $\beta_{m}$ is the $m^{t h}$ symbol from the rate of the $1 / 3$ encoder taking values on the set $\{0,1, \ldots, 7\}, T_{B}$ is the PPM modulation shift (modulation index), $N_{s S}$ is the number of time sub-slots, and $\alpha_{m}$ is the position of the pulse inside the pulse repletion rate taking values on the set $\left\{0,1, \ldots, N_{s s}-1\right\}$. Note that $x(t)$ is a random process.

\section{B. Proposed DLPPM TH-IR System}

Fig. 2 shows the proposed system, allowing comparisons between the conventional and proposed systems based on the DLPPM TH-IR technique. The introduced system differs from the one shown in Fig. 1 by utilising the extra sub-slot stage, which divides each slot by the number of sub-slots for holding new information, leading to an increased bitrate.

Fig. 1 shows the previous system block diagram. We can see that the data coming from the source through the convolutional encoder are used to choose the pulse-position slot of the PPM system. The convolutional encoder's role is to randomize the pulse position for a smooth PSD. The sub-slot position block is used to randomly determine the position of the pulse inside the slot. In other words, this block chooses the sub-slot position for maximum randomness of the positions for consecutive pulses.

In this paper, the position of the sub-slot is used to represent data in order to increase the bitrate and channel capacity. The block diagram of the proposed system is shown in Fig. 2. In this system, the data will be split into two parts each part contains three bits of data: the first part is to go through convolutional encoder- 1 to select the proper slot in the frame, while the second part is to select the proper sub-slot inside the selected slot through convolutional encoder- 2 . This idea will be reflected in (1), where $\alpha_{m}$ on the old system was randomly chosen, and the position means nothing but just helps to randomize the pulse position to obtain a smooth spectrum. Whereas in the proposed system, this value has meaning, representing three bits of data as well as keeping the spectrum smooth.

In general, the PSD of the random TH-IR signal $\bar{X}(f)$ consists of continuous $x(t)$ as well as discrete components [1, 10] and is given by:

$\bar{X}(f)=$

$\frac{1}{T_{r}}|W(f)|^{2}-\frac{1}{T_{r}}|W(f)|^{2}\left(\frac{1}{N_{S} * N_{S S}}+\frac{2}{\left(N_{S} * N_{S S}\right)^{2}} \sum_{n=1}^{\left(N_{S} * N_{S S}\right)-1}\left(\left(N_{S} *\right.\right.\right.$

$\left.\left.\left.N_{s s}-|n|\right) \cos \left(2 \pi f n T_{c}\right)\right)\right)+$

$\frac{1}{T_{r}^{2}}\left(\frac{1}{N_{S} * N_{S S}}+\right.$

$\frac{2}{\left(N_{S} * N_{S S}\right)^{2}} \sum_{n=1}^{\left(N_{S} * N_{S S}\right)-1}\left(\left(N_{s} * N_{S S}-\right.\right.$

$$
\left.\left.|n|) \cos \left(2 \pi f n T_{c}\right)\right)\right) \sum_{-\infty}^{\infty} \delta\left(f-\frac{k}{T_{r}}\right)
$$

where $W(f)$ is the Fourier transform of $w(t)$ and $N_{s}$ is the number of time slots. The value of $T_{B}$ is chosen to eliminate as many spectral lines as possible [11]. The proposed system is a new modulation technique based on conventional DLPPM TH-IR [6]. The proposed DLPPM TH-IR scheme maximizes the average separation between modulated pulses to achieve greater resistance to large delay spreads. In addition, the proposed DLPPM TH-IR randomizes the time offset between adjacent pulses to provide greater immunity to multiple access interference. Thus, the bandwidth efficiency of UWB communications systems is increased.

Fig. 3 is an illustration of an exemplary signal, in the time domain, modulated using the DLPPM TH-IR technique. The signal consists of a number of symbols, having a symbol period $T_{r}$. Symbols are transmitted with very short pulses. Each symbol represents $M$ bits of binary information and has a value in the range of 0 to $2^{\mathrm{M}}-1$. Each symbol is divided into a number of slots, each having a duration equal to $T_{B}$. Each slot contains sub-slots, each having a duration equal to $T_{B} / N_{S S}$. For instance, if $N_{S}=8$ and $N_{s S}=8$, there are 8 slots each containing 8 sub-slots, which correspond to the symbol values $\{0,1,2, \ldots, 7\}$. Finally, each slot is divided into $T_{B} / 8$ time sub-slots, which correspond to the conventional DLPPM THIR code sequence.

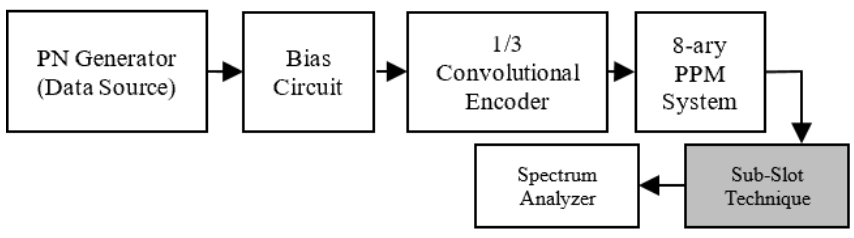

Fig. 1. Conventional DLPPM TH-IR System Block Diagram [6]. 


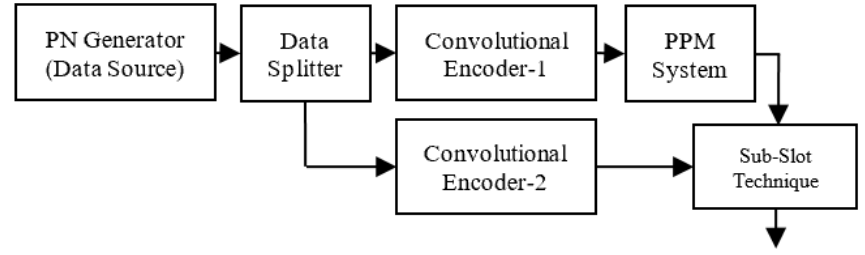

Fig. 2. Proposed System Block Diagram using DLPPM TH-IR Technique.

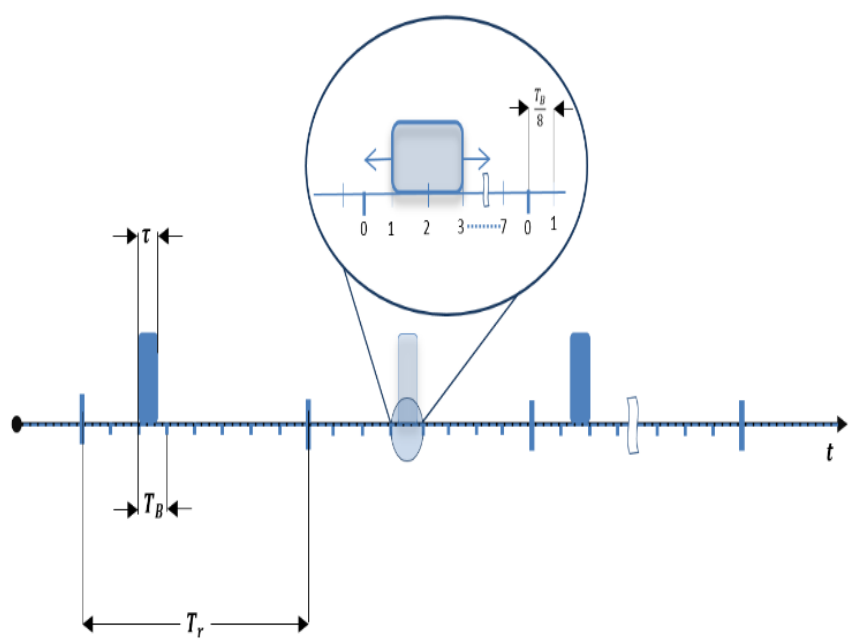

Fig. 3. An Illustrative Example of the Proposed DLPPM TH-IR Signal, where $N_{S}=8$ and $N_{S S}=8$.

Comparison between the conventional and proposed systems is described again in the signal shown in Fig. 3. This signal represents three bits of data when used in the old system, which exactly represents the data " 011 " in this example, as the pulse is in the third slot, where one frame of $T_{r}$ has eight slots. As a result, moving this pulse inside the slot has one main function, which is to achieve maximum randomness and smooth PSD. In the proposed system, the signal can be used to represent six bits of data with no effect on the PSD smoothness. For instance, this signal represents the data " 011001 ", where the three bits on the left " 011 " represent the slot position exactly as they do in the old system, and the other three bits on the right " 001 " represent the subslot position, with this position determined from the (1/3) convolutional encoder- 2 .

\section{ANALYTICAL AND Simulation RESUltS}

\section{A. Analytical Results}

In this section, we compare a conventional DLPPM TH-IR with the new proposed DLPPM TH-IR, where $T_{r}=8 \times 10^{-9} \mathrm{sec}$, $N_{s}=8$, and $R W B=20$, with $40 \mathrm{GHz}$ applied and tested for both cases. Different levels of $N_{s}$ have been selected for the DLPPM TH-IR system to detect the effects on PSD.

Fig. 4 shows the theoretical PSD obtained by using the conventional PPM TH-IR system described in [6] for Resolution Bandwidth (RBW) equal to 20 and $40 \mathrm{GHz}$. It is evident that the tested signal had both random-like and harmonic-like components. Fig. 4 shows that the PSD section consists of 10 spectral lines. These spectral lines do not comply with regulatory spectral masks such as the one used by the FCC. When the RBW increases from 20 to $40 \mathrm{GHz}$, as shown in Fig. 4(b), it is clearly seen that the displayed power of the spectral lines does not change. However, the level of the continuous-like component increases by about $8 \mathrm{dBm} / \mathrm{MHz}$.

Fig. 5 shows theoretical PSDs obtained by using the conventional and proposed DLPPM TH-IR systems described in Section 2 and depicted in Fig. 1 and 2. In Fig. 5(a), the DLPPM TH-IR system has 8 slots $\left(N_{s}\right)$, and each slot has been divided into 4 sub-slots $\left(N_{S S}\right)$. Fig. 5(a) consists of spectral lines repeated every $4 \mathrm{GHz}$, with the total number of spectral lines reduced and smooth harmonics components achieved compared to the conventional PPM TH-IR system, as shown in Fig. 4(a).

Fig. 5(b) shows the PSD section consisting of spectral lines repeated every $8 \mathrm{GHz}$. Fig. 5(c) shows the PSD section consisting of spectral lines repeated every $11 \mathrm{GHz}$, with the total number of spectral lines reduced and smoother harmonics components achieved. In the last case, Fig. 5(c), the spectral line is outside the UWB legalizing spectrum across $7.5 \mathrm{GHz}$, between 3.1 and $10.6 \mathrm{GHz}$ [12], which provides an interference-free system. Identical responses have been achieved for both systems, as expected.

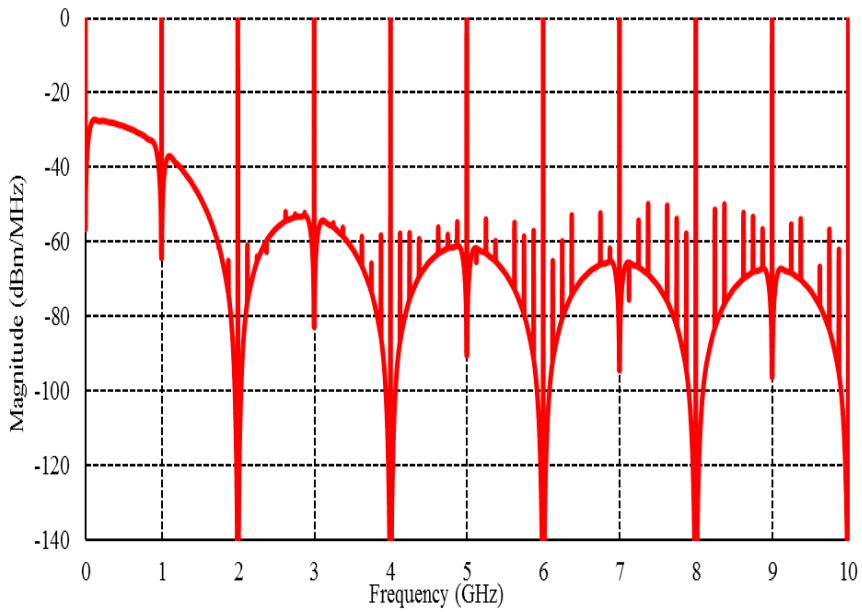

(a) $T_{r}=8 \times 10^{-9} \mathrm{sec} ; N_{s}=8 ; R W B=20 \mathrm{GHz}$.

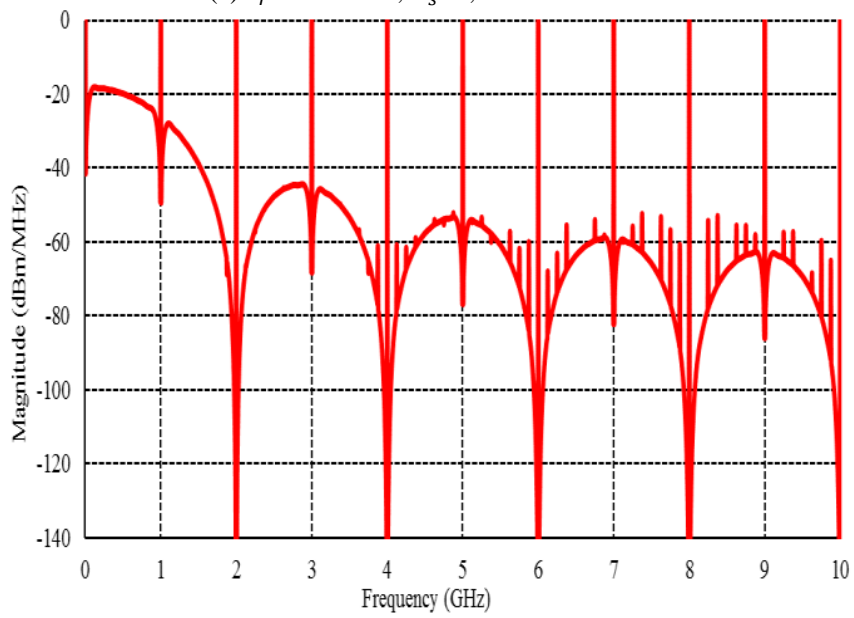

(b) $T_{r}=8 \times 10^{-9} \mathrm{sec} ; N_{s}=8 ; R W B=40 \mathrm{GHz}$.

Fig. 4. Estimated PSDs Performed with different Resolution Bandwidths (RBWs) from [6] 


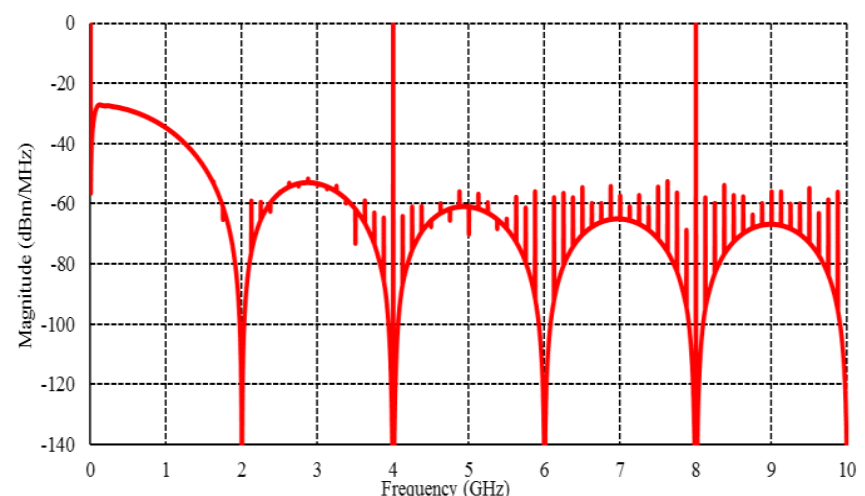

(a) $T_{r}=8 \times 10^{-9} \mathrm{sec} ; N_{s}=8 ; N_{S S}=4 ; R W B=20 \mathrm{GHz}$.

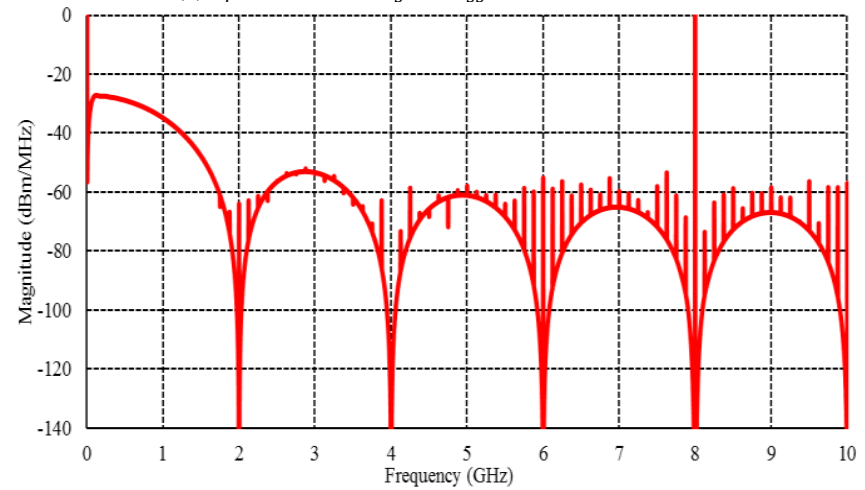

(b) $T_{r}=8 \times 10^{-9} \mathrm{sec} ; N_{s}=8 ; N_{S S}=8 ; R W B=20 \mathrm{GHz}$.

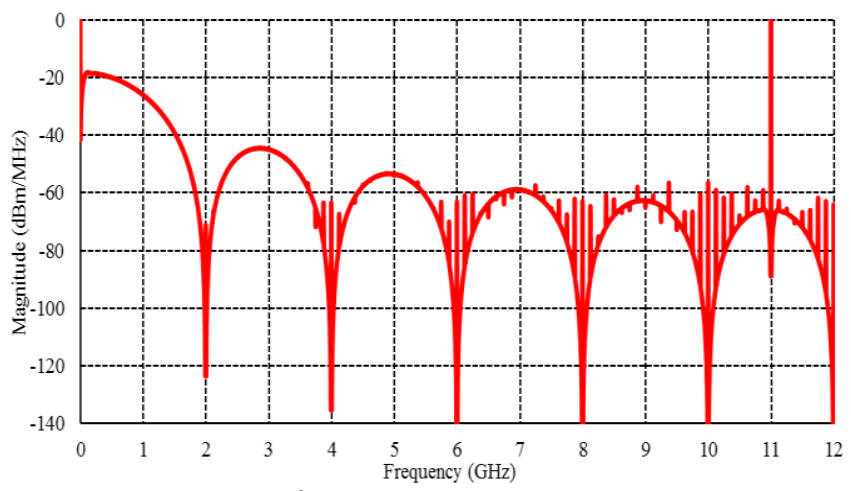

(c) $T_{r}=8 \times 10^{-9} \mathrm{sec} ; N_{S}=8 ; N_{S S}=11 ; R W B=40 \mathrm{GHz}$.

Fig. 5. Estimated PSDs Obtained by an Analytical Model with different RBWs and $N_{s s}$ (for Both Systems).

\section{B. Simulation Model Results}

The conventional and proposed DLPPM TH-IR systems, shown in Fig. 1 and 2, respectively, have been built inside Simulink/MATLAB in order to compare the two systems for bitrate, as well as the selected code for eliminating the spectral lines or increasing smoothness to reduce the interference problem on other users. In the simulation system, each frame has eight slots and each slot has eight sub-slots. Both systems have same bandwidth but the one depicted in Fig. 1 represents three bits and the one depicted in Fig. 2 represents six bits. As a result, a doubled bitrate is expected to be achieved by this technique (proposed system).

1) PSD: In this section, a [ $\left.\begin{array}{lll}4 & 2 & 1\end{array}\right]$ code set of systematic convolutional codes with rate $1 / 3$ has been applied and tested with equal probability [13-15]. The same process has been repeated to detect the difference between the conventional and proposed DLPPM TH-IR systems.

The results for both systems are compared for the same metrics: Pseudo Noise (PN) length, convolutional encoder codes, symbol rate, pulse shape $(0.5 \mathrm{~ns})$, and with equal probability. In the DLPPM TH-IR system, each slot $\left(N_{s}\right)$ is divided into 8 sub-slots $\left(N_{s S}\right)$. The pulse position in both systems will be determined in the same way, with the only difference being the location of the pulse inside the slot. In both (conventional/proposed) DLPPM TH-IR systems, a convolutional encoder is used to decide the sub-slot pulse position. In the old system, the sub-slot position is randomly chosen by the (Sub-slot Technique) block, as shown in Fig. 1. In the new system, part of the data came from the source is used to choose the sub-slot position to represent three bits of data.

Fig. 6 shows an excellent PSD performance obtained by using conventional vs proposed DLPPM TH-IR systems with the same conditions. Excellent performance returns to the randomness between consecutive pulses. Furthermore, it can reduce the spectral lines or make them smoother. Thus, the proposed system will not affect the contribution achieved in [6], but it will enhance the bitrate as demonstrated in the next sub-section.

2) Bitrate: Fig. 7 shows the receiver side used to detect the data for both systems. In the old system receiver, as shown in Fig. 7(a), the (Slot Location Detector) block can detect which slot has a pulse regardless of which sub-slot is in. Then the signal will go to the PPM demodulator to convert these symbols to three bits encoded data. After that, Viterbi decoder is used for decoding and recovering the original data. In the new system receiver, as shown in Fig. 7(b), the (Slot/Sub-slot Location Detector) block is used to separate the information bits into two parts: the first part is comprising the bits that control the location of the slot and the second part is comprising the bits that specify the sub-slot. The slot location will be processed by the upper side of the receiver exactly as the old system, and the sub-slot by the lower side. The data from both sides are combined by (Data Combiner) block. The overall result is six bits of data received per one PPM frame instead of three bits per frame in the old system.

The easiest way to demodulate a PPM signal is to use a decoder. This is one of the best techniques for digital communications when computational complexity dominates in importance. It permits major equipment simplification while obtaining the full performance benefits of maximum likelihood decoding. The decoder structure is relatively simple for a short constraint length $N$, making decoding feasible at relatively high rates of up to $10 \mathrm{Gbit} / \mathrm{s}$ [16].

Simulation has been run for $20 \mu s$ for each system, where the data source (PN) sampling rate is $4 n s$ for the old system and 2 ns for the new one. The channel assumed to be noiseless. The number of bits received in the old system is 4801, as shown in Fig. 8(a) whereas the proposed system has received 9801 as shown in Fig. 8(b). The number of delayed bits is 200. By adding 200 to each system, the total bits 
received for the old and the proposed systems are 5001 and 10001 , respectively, where both systems have a bandwidth of $2 \mathrm{GHz}$. Obviously, we can see that the bitrate has been duplicated exactly. The noiseless channel capacity is shown in (3) [17]:

Maximum Bitrate $=2 * B W * \log _{2}(L)$

where $B W$ is the bandwidth and $L$ is the number of levels, which is eight for the conventional system and 64 for the proposed system.

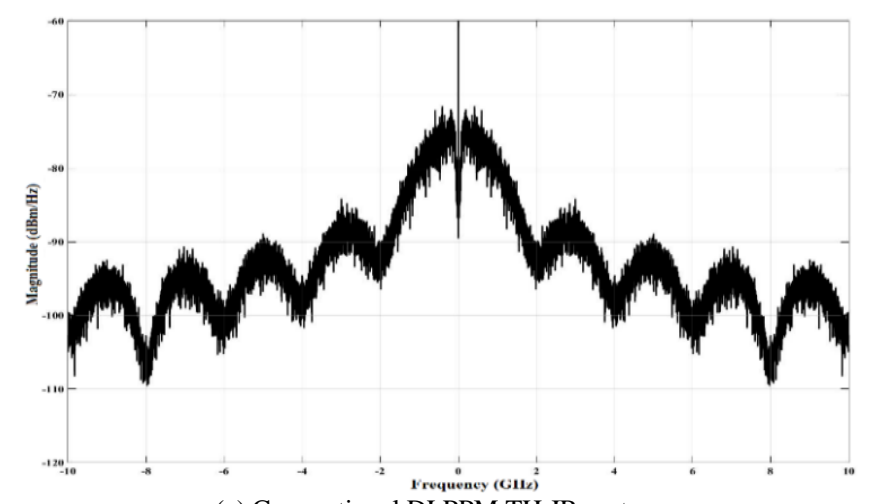

(a) Conventional DLPPM TH-IR system.

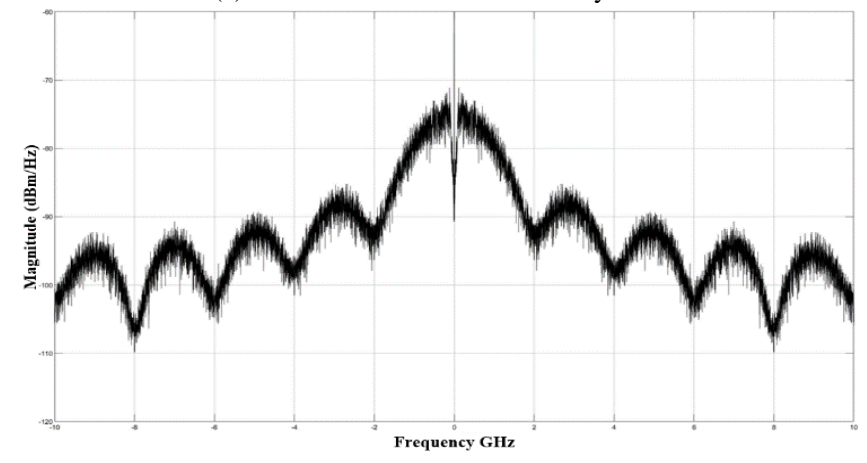

(b) Proposed DLPPM TH-IR system.

Fig. 6. PSD with Equal Probability for a Systematic Code [4 2 1].

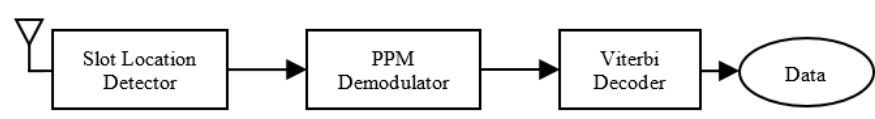

(a) Conventional DLPPM TH-IR System.

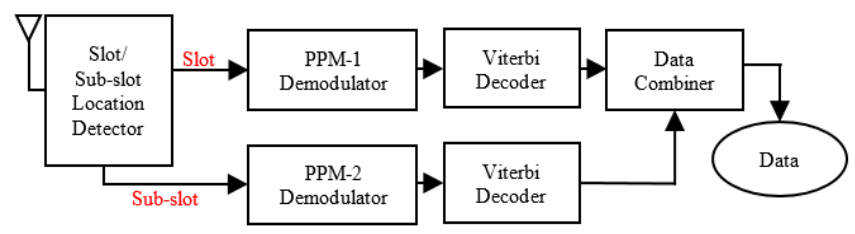

(b) Proposed DLPPM TH-IR System.

Fig. 7. Block Diagram of the Receiver for both Systems.

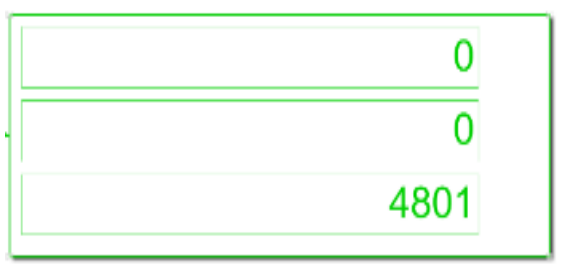

(a) Conventional DLPPM TH-IR System.

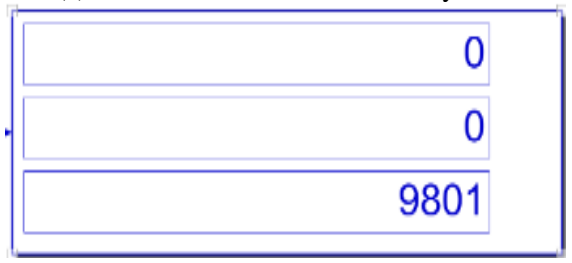

(b) Proposed DLPPM TH-IR System.

Fig. 8. Bitrate Counter based on Receiver Built Inside Simulink/MATLAB.

\section{CONCLUSION}

The present method provides a novel technique which meets the requirements described earlier with the help of a DLPPM TH-IR scheme that allows for a greater throughput than the conventional DLPPM TH-IR scheme. This can be achieved by maximizing the pulse separation and randomizing the time offset between pulses in a time-efficient manner to ensure the period is maximized.

A mathematical representation of the PSD of a DLPPM TH-IR UWB signal was derived. The analytical result was used to investigate the effect of the variable position of pulses in the DLPPM TH-IR system in the PSD of the signal, and it was found that it can be effectively used to eliminate some spectral lines or to diminish the peak value of the PSD. It has been observed that DLPPM TH-IR significantly outperforms conventional PPM TH-IR with respect to spectral efficiency when the location of a pulse is variable within each slot. The hardware complexity at the receiver side does not need to be increased, which makes the DLPPM scheme very attractive for TH IR-UWB communication systems.

Theoretically, the bitrate can be increased without limit by increasing the number of sub-slots. As a result, the Inter Symbol Interference (ISI) will be increased. This can be clearly noted when the phase error is added to the old and proposed systems. The old system is less affected than the new one. This can be explained as follows: in the old system, any change in the sub-slot position of the pulse will not affect the symbol data for one frame of the PPM signal. It is sufficient to detect the pulse inside the same slot to avoid ISI. In contrast, in the proposed system, a small phase can lead to an error of three bits of six bits for one frame of data.

Finally, the simulated results within Simulink/MATLAB were compared to the conventional DLPPM TH-IR UWB. The results showed that when testing with a systematic code [4 2 1], a smoother PSD without spectral lines and a doubled bitrate were achieved. 


\section{REFERENCES}

[1] Villarreal-Reyes, R. M. Edwards and B. Al-haj Moh'd, "On for the Comparison of Measurement and Simulation of the Power Spectral Density of PPM TH-IR UWB Signals,' Loughborough Antennas and Propagation Conference Proceedings, LAPC 2006, March 2006, pp.118122.

[2] Win, M. Z., Scholtz, R. A.: Ultra-Wide Bandwidth Time Hopping Spread-Spectrum Impulse Radio Wireless Multiple Access Comm. IEEE Trans On Comm, vol. 48, pp. 679-691, (2000).

[3] Scholtz, R. A.: "Multiple Access with Time-Hopping Impulse Modulation," In: Proc. MILCOM 1993, Bedford, MA, pp. 447-450, (1993).

[4] T. Ballal and T.Y. Al-Naffouri, "Low-sampling-rate ultra-wideband channel estimation using equivalent time sampling," Signal Processing, IEEE Transactions on, vol. 62, no. 18, pp. 4882-4895, Sep. 2014.

[5] Das, B., G. K. Mishra, S. Kanungo, and B. K. kumar Sahu. "Performance improvement through interference mitigation techniques in Transmitted Reference UWB system used in WPAN overlay systems." (2015).

[6] Qasem, Nidal, and Bashar Al-Haj Moh'd. "Enhancing the Power Spectral Density of PPM TH-IR UWB Signals Using Sub-Slots Technique." International Journal of Computer Science and Network Security (IJCSNS) 17, no. 1 (2017): 124.

[7] S. Villarreal-Reyes and R. M. Edwards, "On the Behaviour of Simulation-DFT Based Analysis for Spectral Estimation of PPM TH-IR UWB Signals," Loughborough Antennas and Propagation Conference Proceedings, LAPC 2005, April 2005.
[8] Yajnanarayana, Vijaya, Satyam Dwivedi, Alessio De Angelis, and Peter Händel. "Spectral efficient IR-UWB communication design for low complexity transceivers." EURASIP Journal on Wireless Communications and Networking 2014, no. 1 (2014): 1-13.

[9] Marshall, Alan G., and Francis R. Verdun. Fourier transforms in NMR, optical, and mass spectrometry: a user's handbook. Elsevier, 2016.

[10] M. Z. Win, "Spectral density of random UWB signals", IEEE Communications Letters, Vol. 6, No. 12, pp. 526-528, Dec 2002.

[11] S. Villarreal-Reyes and R. M. Edwards, "Spectral Line Suppression in TH-IR Ultra Wideband Systems," $5^{\text {th }}$ IEE International Conference on Mobile Communications Technologies (3G 2004), Oct 2004.

[12] Federal Communications Commission. "Revision of part 15 of the commission's rules regarding ultra-wideband transmission systems, first report and order (ET Docket 98-153)." Adopted Feb 14 (2002): 2002.

[13] R. Johannesson ."Some rate $1 / 3$ and 1/4 binary convolutional codes with an optimum distance profile". IEEE Transactions on Information Theory, 23 no.2(1977): 281-283.

[14] L. Hanzo, TH.Liew, and B. L. Yeap. Turbo coding, turbo equalisation and space-time coding". John Wiley \& Sons, 2002.

[15] Nail, Rana, and Nidal Qasem. "Enhancing the Power Spectral Density of PPM-IR for Ultra-Wide Band signals by using a convolutional encoder." In Internet Technologies and Applications (ITA), 2015, pp. 14-17. IEEE, 2015.

[16] Chase, David. "Code combining-a maximum-likelihood decoding approach for combining an arbitrary number of noisy packets." IEEE transactions on communications 33, no. 5 (1985): 385-393.

[17] Bagad, V. S., and I. A. Dhotre. Computer Networks-II. Technical Publications, 2009. 